\title{
Development of Active Orthosis for Lumbago Relief- Improvement of Pneumatic Textile Actuator for Orthosis -
}

\author{
Shinsaku FUJIMOTO ${ }^{\text {a }}$, Tetsuya AKAGI and Akihiro YAMAMOTO \\ Department of Inteligent Mechnical Engineering, Okayama University of Science, \\ 1-1 Ridaicho, Kita-ku Okayama, JAPAN
}

\begin{abstract}
It is important to develop the orthosis which improves the Quality of Life (QOL) and maintains health conditions. As one of the treatment methods done to lumbago(low back pain), the waist fixation method with the spinal brace or the orthosis is prescribed. A waist active orthosis implemented with pneumatic flexible actuators have been developed. However, several problems of the previous actuator were that the strain and the generated force were small for the orthosis.Thus, this paper proposesthe improved actuator for the orthosis. Theimproved actuator is modeled and the reliability of static and dynamic model is validated through experiment. As a result, it was confirmed that the improved actuator had the strain of 2 times and the generated force of 1.3 times, in comparison with the previous actuator. And the dynamic model including volume of actuatorcould be represented by a second-order form with a dead time.
\end{abstract}

\section{Introduction}

It is important to improve the QOLand maintain the health condition for elderly people. Our special attention is directed to lumbago because many people feel a back pain in daily life. As one of the treatment methods done to lumbago, the waist fixation method[1] with a spinal brace or an orthosis is performed. Generally, an orthosis is wrapped around the waist made of cloth and the rubber textile. However, the existing orthosis have the three difficult and important issues.

1) Problem difficult to meet on-demand requirements2) Problem to suppress blood stream

3) Problem difficult to customize

In order to solve these issues, a waist active orthosis implemented with pneumatic flexible actuators is developed and pressure control method of pneumatic flexible actuators is established. However, the customizing issues have not been solved.In this study, firstly, the prototype of pneumatic textile actuator (hereafter called PTA) has been developed. But several problems of the previous actuator were that the strain and the generated force were small for the orthosis. The previous actuatoris indirectly driven by a McKibbenactuator. Thus, the improved actuator changes to the driving method that can be contracted directly by a silicone tube. Therefore the improved actuator is modeled and the reliability of static and dynamic model is validated through experiment.

\footnotetext{
${ }^{\mathrm{a}}$ Corresponding author : fuji@are.ous.ac.jp
} 


\section{Structure of Improved PTA}

The previous PTA has a structure which inwrought with a long McKibben-type actuator[2] into two soft cloths (See Figure 1(a)). When the compressed air is inject into the supply port, the McKibben-type actuator contracts to an axial direction. As the result, the PTA shrinks indirectlyby the seam constraint. Thus, two problems of the previous PTAwere that the strain and the generated force were small for the orthosis.

On the other hand, the improved PTA has a structure which inwrought with a silicone tube into two soft cloths as shown in Figure 1(b).

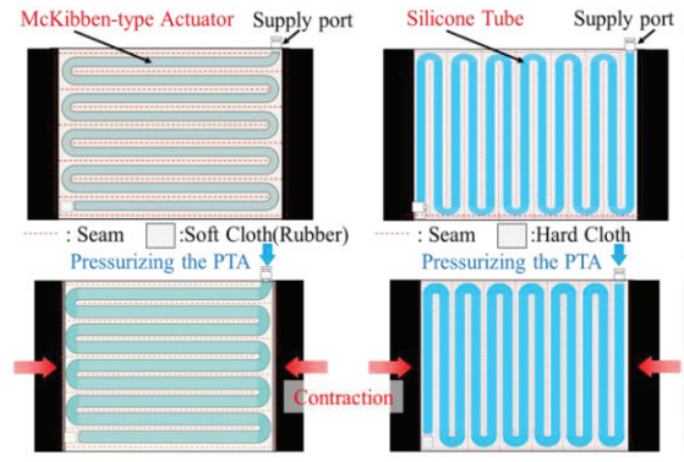

(a) Previous PTA. (b) Improved PTA.

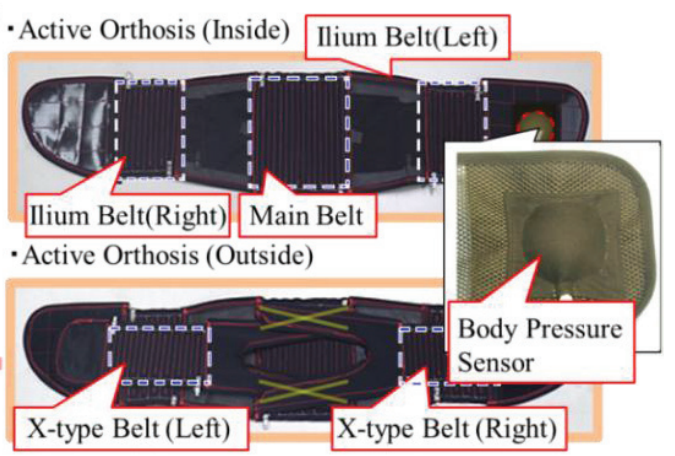

(c) Prototype of Active Orthosis.

Figure 1. Previous PTA and improved PTA.

Figure 1(c) shows the prototype of the active orthosis for the lumbago relief. The proposed active orthosis consists of five PTAs (=belts) and the body pressure sensor. The size of the orthosis is $970 \times 200 \times 7 \mathrm{~mm}$, andthe mass is $300 \mathrm{~g}$. The active orthosis has a double structure. One structure is the body-orthosis that is configured in the main belt and the ilium belts (right and left). Another structure is two X-type belts.

Figure 2(a) shows the operating principle of PTA. When the compressed air is injected into the supply port, the silicone tubeexpands a radial direction. As the result, the PTA shrinks directlyby the constraints of the seam constraint and the hard cloth.

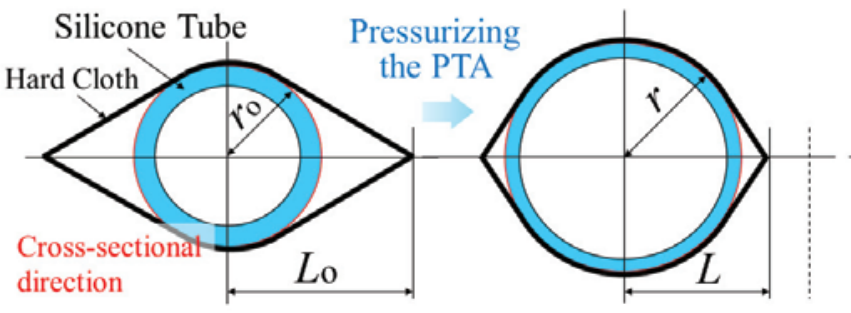

(a)The operating principleof improved PTA.

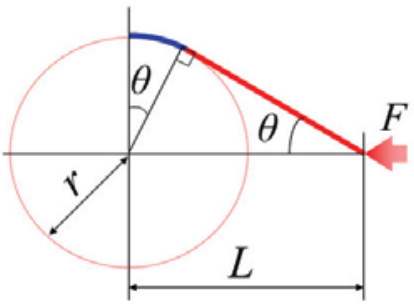

(b) Static model.

Figure 2. The operating principle and static model of improved PTA.

\section{Modeling of Improved PTA}

This chapter will be described about the construction methods of a static model and a dynamic model. And theorthoses control valvesystem is modeled and the reliability of exhaust and supply model is validated through experiment.

\subsection{Static Model of Improved PTA}


From the view of energy conservation, the input work of the improved PTA should equal the output work which a system includes force dissipation or force loss $F_{\text {diss }}$. Suppose that the actuator is in this ideal condition, a following equation of static model holds.

$$
P \cdot d V=-\left(F+F_{\text {diss }}\right) \cdot d L
$$

where $P$ is the internal gauge pressure, $d V$ is the volume change, $F$ is the force generated by the PTA, and $d L$ is the axial displacement, respectively.

When the clothes of PTA are made with the material which can't shrink, the half pitch length $b$ of the seam doesn't change. The outside radius $r$ of the silicone may be expressed as

$$
r=b(\theta+\cot \theta)^{-1}
$$

where $\theta$ is the angle between the seam axis and the silicone (See Figure 2(b)).

The inner volume $\mathrm{V}$ of silicone is given by

$$
V=l \cdot \pi r_{S}^{2}
$$

where $r_{\mathrm{S}}$ is the inside radius and $l$ is length of the silicon, respectively.

Assume that the outside radius $r$ change but the cross-sectional area $S_{\mathrm{O}}$ and the length $l$ of silicone don't change. The following equation is expressed by

$$
V=l\left(\pi r^{2}-S_{O}\right) \because S_{O}=\pi\left(r^{2}-r_{S}^{2}\right)=\text { Const. }
$$

Eq.(2) is substituted for eq.(4). Then, the inner volume $V$ can be rewritten as

$$
V=l\left\{\pi b^{2}(\theta+\cot \theta)^{-2}-S_{O}\right\}
$$

On the other hand, since the force term $F+F_{\text {diss }}$ of eq.(1) is given by

$$
F+F_{\text {diss }}=-P \frac{d V}{d L}=-P \frac{\partial V}{\partial \theta} \cdot \frac{\partial \theta}{\partial L}
$$

Hence, the generated force $F$ is

$$
F=2 \pi l b P\left\{\frac{\cos \theta}{\theta(\theta+\cot \theta)}\right\}-F_{d i s s}
$$

where each partial differentiation with eq.(6) is

$$
\frac{\partial V}{\partial \theta}=\frac{2 \pi l b^{2} \cot ^{2} \theta}{(\theta+\cot \theta)^{3}}, \frac{\partial L}{\partial \theta}=-\frac{b \theta \cos \theta}{(\theta \sin \theta+\cos \theta)^{2}}
$$

The length $L$ is the involute function with respect to $\theta$. Thus, the angle $\theta$ is the nonlinear inverse-function with respect to $L$. However, the inverse function of an involute function can't be solved analytically. In this paper, this inverse function will be solvednumerically.

Therefore, to solute the nonlinear function $f(\varepsilon)$ that is the first term of right side in eq.(7), the relation between the function $f(\varepsilon)$ and the strain $\varepsilon$ is calculated by the numerical solution such as Newton method.

$$
f(\varepsilon)=\frac{\cos \theta}{\theta(\theta+\cot \theta)} \because \varepsilon=\frac{L-L_{O}}{L_{O}}
$$

where $L_{\mathrm{O}}$ denotes an initial length of PTA. 


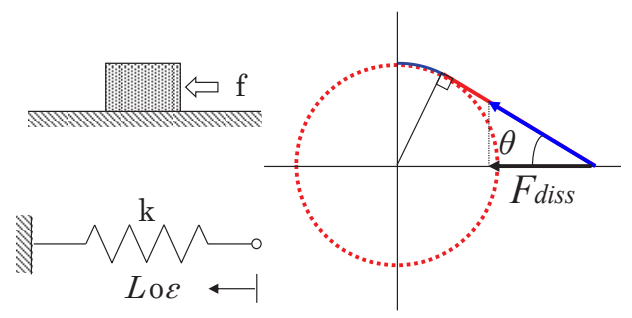

Figure 3. Concept of dissipation force $F_{\text {diss }}$.

If a strain of the PTA is held in less than 25 percent, the function $f(\varepsilon)$ with respect to strain $\varepsilon$ is approximated by following first-order function.

$$
f(\varepsilon)=\frac{\cos \theta}{\theta(\theta+\cot \theta)} \equiv \alpha \cdot \varepsilon+\beta
$$

where $\alpha$ and $\beta$ are characteristics parameters of PTA.

Eq.(10) is substituted for eq.(7). The generated force $F$ is given by

$$
F=2 \pi l b P(\alpha \cdot \varepsilon+\beta)-F_{\text {diss }}
$$

Then, the dissipation force is discussed. In this paper, the dissipation force $F_{\text {diss }}$ includes a static frictional force and the force loss of an elastic force which occur to the silicone and the cloth. Figure 3 shows the concept of dissipation force $F_{\text {diss }}$.

The static model of the improved PTA with the dissipation force is expressed by

$$
F=2 \pi l b P(\alpha \cdot \varepsilon+\beta)-\left(k L_{O} \varepsilon+f\right) \cos \theta
$$

where $k$ is an elastic coefficient and $f$ is a static frictional force.

Furthermore, we discuss the relation between strain $\varepsilon$ and cosine function that is the second term of right side in eq.(12). If a strain of the PTA is held in less than 20 percent, the function $\cos (\theta)$ with respect to strain $\varepsilon$ is approximated by following first-order function.

$$
\cos \theta \equiv \gamma \cdot \varepsilon+\eta
$$

where $\gamma$ and $\eta$ are the approximate coefficients of the cosine function.

Eq.(13) is substituted for eq.(12). Therefore, the static model of the improved PTA is given by

$$
F=P\left(\alpha_{1} \varepsilon+\alpha_{0}\right)-\left(\beta_{2} \varepsilon^{2}+\beta_{1} \varepsilon+\beta_{0}\right)
$$

where coefficients $\alpha_{1}=2 \pi l b \alpha, \alpha_{0}=2 \pi l b \beta, \beta_{2}=k L_{\mathrm{o}} \gamma, \beta_{1}=k L_{\mathrm{o}} \eta+f \gamma$ and $\beta_{0}=f \eta$ are PTA characteristics constants, respectively. Clearly, the force $F$ depends on both pressure $P$ and strain $\varepsilon$. When pressure increases, the silicone tube expands radially and PTA shortens in length to generate a radial contraction force.

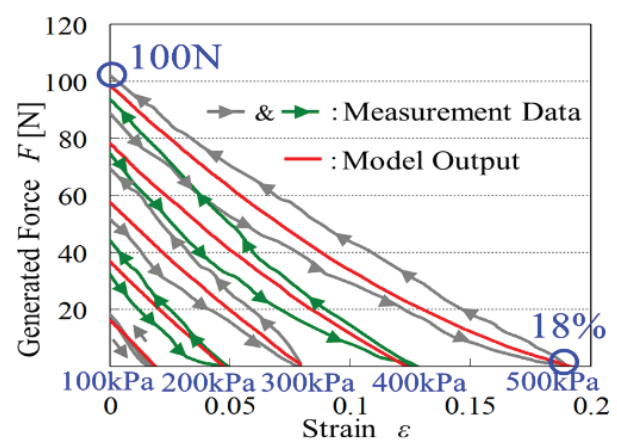

Figure 4.Varification experiment of static model (Results of measurement and model output signals). 
In order to prove versatility of equation (14), the comparison was done between the measurement data and force model. It can be seen in Figure 4 that both generated force Fand strain sincrease with pressure. The static force model of the improved PTA is that it is located in the average of the hysteresis loop. Therefore, the accurate fitting is demonstrated in Figure 4. And ,The improved actuator had the strain performance of 2 times and the generated force of 1.3 times, in comparison with the previous actuator.

\subsection{Three Modes of Tested Valve}

Figure5 shows the operating principle of the orthosis controlvalve. The tested valve consists oftwo on/off-type control valves (SMC Co. Ltd.,S070C- SDC-32) that both output ports are connected each other. One valveis used as the supply valve, and another is used as the exhaust valve. Two valves can adjust output flowrate like a variable fluid resistance by means of the fast switching. The size of the on/off valve is $36 \times 14.5 \times 7.2 \mathrm{~mm}$, andthe mass is $5 \mathrm{~g}$. The total mass of the orthosis control valve including the controller (Micro-computer: Renesas Co. Ltd., H8/3664F) is very light, that is about $220 \mathrm{~g}$. The orthosis controlvalve has three modes (See Figure 5(a),(b) and (c)) as follows:

(a) Supply Mode: The exhaust valve is the off state. And, the pressure of the tank (PTA) can be adjusted by the fast switching of the supply valve.

(b) Exhaust Mode: Conversely, the supply valve is the off state. And, the pressure of the tank (PTA) can be adjusted by the fast switching of the exhaust valve.

(c) Hold Mode: When both valves are the off states, the pressure of the tank (PTA) is kept a constant pressure.

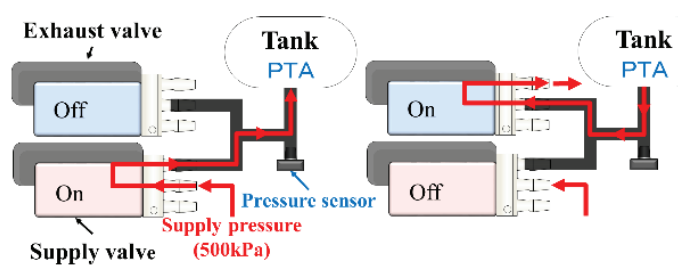

(a) Supply Mode

(b) Exhaust Mode

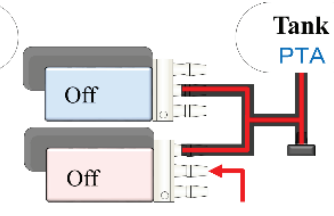

(c) Hold Mode

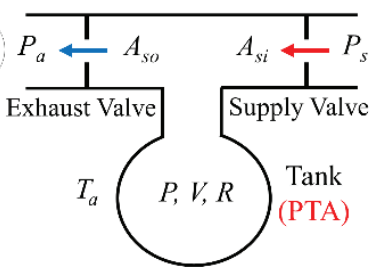

(d) Analytical model

Figure 5. Three Modes and analytical modelof the orthosis controlvalve.

\subsection{Dynamic Model of Improved PTA[3] [4]}

Figure 5(d) shows the analytical model of the orthosis control valve. The mass flow rate of supply valve $Q_{i}$ and the exhaust valve $Q_{o}$ are given as follows.

$$
\begin{array}{r}
Q_{i}=A_{s i} P_{s} \sqrt{\frac{2}{R T_{a}}} g\left(z_{i}\right), \quad z_{i}=\frac{P}{P_{s}}: \text { Supply State } \\
Q_{o}=A_{s o} P_{a} \sqrt{\frac{2}{R T_{a}}} g\left(z_{o}\right), \quad z_{o}=\frac{: P_{E}}{P} \text { xhaust State }
\end{array}
$$

where $R$ and $T_{a}$ mean a gas constant and an absolute temperature, respectively. The function $g(z)$ that expresses the state of flow is given as follows.

$$
\begin{aligned}
& g(z)=\sqrt{\frac{\kappa}{\kappa-1}\left(z^{2 / \kappa}-z^{(\kappa+1) / \kappa}\right)} \quad[0.528 \leq \mathrm{z} \leq \mathrm{l} \text { Subsonic Flow } \\
& g(z)=\sqrt{\frac{\kappa}{\kappa+1}\left(\frac{2}{\kappa+1}\right)^{2 /(\kappa-1)}} \quad[0 \leq z \leq 0.528] \text { Choked Flow }
\end{aligned}
$$


where $\kappa$ means a specific heat ratio $(=1.4)$. The pressure $P$ in the volume $V$ of PTA is given by next equation.

$$
\begin{aligned}
& \dot{P}=\frac{d P}{d t}=\frac{\kappa R T_{a}}{V}\left(Q_{i}\right) \quad: \text { Supply State } \\
& \dot{P}=\frac{d P}{d t}=\frac{\kappa R T_{a}}{V}\left(-Q_{o}\right) \quad: \text { Exhaust State }
\end{aligned}
$$

The systemsof Eqs.(19) and (20) are non-linear systems with respect to the input of sectional area $A_{\mathrm{s}} *^{*}=\mathrm{i}$ or $\left.\mathrm{o}\right)$. Here, the simple idea is to approximate a non-linearsystem by a linear one (around the pressure point $P=P_{E}(z=0.528)$ )

$$
\begin{array}{lll}
\dot{x}=-\frac{2.70 \times 10^{2} \cdot A_{s o}}{V} x-\frac{4.61 \times 10^{4}}{V} v & \because x=P_{E}-P, v=A_{o} & : \text { Supply State } \\
\dot{x}=-\frac{2.78 \times 10^{2} \cdot A_{s i}}{V} x+\frac{4.68 \times 10^{4}}{V} v & \because x=P_{E}-P, v=A_{i} \quad: \text { Exhaust State }
\end{array}
$$

In the linearization, the atmosphericpressure $P_{a}$ of $101.3 \mathrm{kPa}$, the room temperature $T_{a}$ of $298 \mathrm{~K}$, the gas constant $R$ of $287 \mathrm{~J} / \mathrm{kg} / \mathrm{K}$, the supply pressure $P_{s}$ of $500 \mathrm{kPa}$ were used. And the sectional area of the supply port $A_{s i}\left(=2.02 \times 10^{-7} \mathrm{~m}^{2}\right)$ and thearea of exhaust port $A_{s o}\left(=2.30 \times 10^{-7} \mathrm{~m}^{2}\right)$ were adopted the values of catalog specification (on/off valve). The obtained linear system can be expressed by the first-order transfer function. The pole of first-order system depends on the volume $V$ of PTA. The smallervolume of PTA, the faster response speed becomes.

On the other hand, suppose that the sectional area $=A *$ of the on/off valve is opened (or closed) slowly. Suppose that the switching area $A_{*}(*=\mathrm{i}$ or o $)$ of valve is approximated by dead time $L(=3$ $\mathrm{ms})$ and time constant $T_{\mathrm{o}}(=2 \mathrm{~ms})$ of a primary delay system.

$$
v(s)=A_{*}(s)=\frac{A_{s^{*}}}{T_{o} s+1} \cdot e^{-L s} \cdot u(s) \quad \begin{cases}u(s)=1 & : \text { Supply Mode } \\ u(s)=-1 & : \text { Exhaust Mode } e_{\text {New Input (23) }} \\ u(s)=0 & : \text { Hold Mode }\end{cases}
$$

From Eqs.(21)-(23), the transfer function of on/off valve system with volume of PTA is given by a second-order form and a dead time.The transfer function of the control valve system with volume of PTA is given by a second-order form with a dead time[5].To validate the reliability of the linear model, the verification experiment of supply $(500 \mathrm{kPaG})$ and exhaust $(0 \mathrm{kPaG})$ motion was performed on conditions of different volumes. Figure 6(a) shows the result (bode diagram) of system identification using the experimental device. And,Figure 6(b) shows the experimental result and output of the proposed model at the volume $V=7 \mathrm{ml}$. From Figure6(b), it can be seen that the output results (rise action and fall action) using the proposed model agree well with the experimental result.The proposed model was a very simple model, but it could be confirmed that the actual valve system including volume can be represented by means of a second-order form with a dead time.

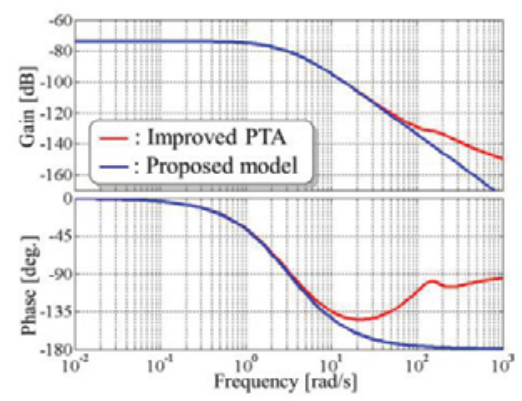

(a)Bode diagramof valve system.

Figure 6. Results of verification experiment.

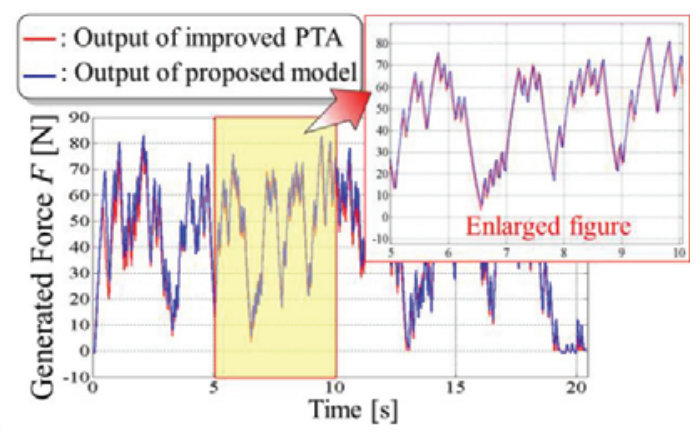

(b) Experimental results of dynamic model 


\section{Conclusions}

This study was aimed to develop the PTA of the orthosis for lumbago relief and the resultingknowledges are summarized as follows:

1) The improved PTAwas modeled and the reliability of static and dynamic model was validated through experiment.

2) The improved actuator had the strain performance of 2 times and the generated force of 1.3 times, in comparison with the previous actuator.

3) The dynamic model including volume of PTA could be represented by means of a secondorder form with a dead time.

\section{Acknowledgments}

The authors would like to acknowledge financial support for this workprovided by QOL (Quality of Life) Innovative Research (2012-2016)from the Ministry of Education, Culture, Sports, Science and Technology of Japan.

\section{References}

1. S. Schulte, H. Rossler, P. Ziehe and F. Higuchi:Bracing and supporting of the lumbar spine, Prostheses and Orthotics International, 6, 3,139-146(1982)

2. H. F. Schulte: The characteristics of the McKibben Artificial Muscle, The Application of External Power in Prosthetics and Orthotics, National Academy of Sciences-National Research Council,94-115(1961)

3. R. B. van Varseveld and G. M. Bone: Accurate Position Control of a Pneumatic Actuator Using On/Off Solenoid Valves, IEEE/ASME Transactions onmechatronics, 2,3, 195204(1997)

4. Y.Moriwake,T. Akagi, S.Dohta and F.Zhao: Development of Low-cost Pressure Control Type Quasi-servo Valve UsingEmbedded Controller,International Symposium on Robotics and Intelligent Sensors, 493-500(2012)

5. N.Abe: The Limiting Optimal Regulator for Systems with Time-delay Considering Disturbance Decoupling, Mem. Inst. Sci., Tech., Meiji Univ., 39, 1,1-6 (2000) (in Japanese) 